\title{
Revisit and insight into the dilemma of strong and brittle materials: Toughening by Grain Boundary Engineering (GBE)
}

\author{
T. Watanabe ${ }^{1 *}$, S. Kobayashi ${ }^{2}$ \\ ${ }^{1}$ Key Laboratory of Anisotropy and Texture of Materials, Northeastern University, Shenyang, China, \\ formerly Tohoku University, Sendai, Japan \\ ${ }^{2}$ Division of Mechanical and Electrical Engineering, Department of Innovative Engineering, Faculty of Engineering, \\ Ashikaga Institute of Technology, Ashikaga, Japan
}

Received 2 March 2015, received in revised form 2 March 2015, accepted 5 April 2015

\begin{abstract}
There is a long pending dilemma, "strong and brittle" materials that still remains unsolved in materials development based on the discipline of Materials Science Engineering (MSE). To solve the dilemma a new concept of "Grain Boundary Engineering" (GBE) was proposed by one of the authors in the early 1980s. In recent years, GBE for toughening of brittle materials has been challenged by several groups including ours, drawing an increasing interest of many researchers. Extensive works have been successfully performed in order to produce "strong and tough" polycrystalline materials. In this article, we present the current progress in GBE, to confirm the situation to what extent the problem has been solved. We pay our special attention to two kinds of brittle materials, i.e. intrinsically and extrinsically brittle ones. These brittle materials can be transformed into "strong and tough" materials by GBE through controlling intergranular fracture under static and cyclic stressing fatigue conditions.
\end{abstract}

K e y word s: brittle materials, Grain Boundary Engineering (GBE), intergranular fracture, grain boundary microstructure, strong and tough materials

\section{Introduction}

\subsection{The dilemma of "Strong and Brittle" materials}

We can make anything beneficial when it is under our control, but it becomes harmful when beyond our control. Even fracture phenomenon can be beneficial, e.g. on a small scale to the machining and shaping of structural components. On the contrary, when fracture occurs beyond our control, as earth-quake on a large scale, it brings about serious damage and disaster to the activities and safety of our human society. It is evident that there is always a strong demand for the control of fracture phenomena occurring in materials world as well as the human world. When we look at the materials world, fracture control research is extremely important to our human society. We materials scientists and engineers have been deeply involved in this important research area, and have a mission to ob- tain full understanding and controlling fracture phenomena occurring in real materials world. The control of fracture is the ultimate goal of fracture research, with much expectation of its beneficial applications.

Despite current fundamental knowledge of microstructure-related properties of engineering materials based on the discipline of modern Materials Science and Engineering (MSE), there is a long standing serious problem concerning bulk mechanical properties of polycrystalline materials, namely, "Strong and Brittle Materials" [1-5]. When the strength of a material is drastically increased by some strengthening mechanism, its ductility and fracture toughness tend to decrease due to dominant fracture of grain boundaries (GBs). This can be seen in almost all the cases of high strength steels, microfine-grained and nanocrystalline metallic materials produced by recently developed processing methods, following the traditional way of the strengthening discipline by grain refinement. This is a long pending dilemma, that is "Strong

*Corresponding author: e-mail address: tywata@fk9.so-net.ne.jp 
and Brittle Materials", as a general feature of mechanical properties of existing polycrystalline metallic, intermetallic and ceramic materials.

This problem has often hindered us from development of high performance structural engineering materials. Accordingly, in order to obtain some useful suggestion and insight to solve the problem, we need to confirm the current situation of the progress in our fracture research field. Full understanding of fracture processes and mechanisms responsible in brittle fracture is urgent from macroscopic, microscopic and atomistic points of view. It is well known that severe brittleness of polycrystalline materials is due to the occurrence of dominant intergranular fracture so that we need to look for and find a possible way to control brittle intergranular fracture. Firstly we must recognize that there are two types of brittle materials, i.e. intrinsically brittle materials and extrinsically brittle materials embrittled by extrinsic origins.

\subsection{Question to conventional strengthening by grain refinement}

Let us consider the physical origin of effects of GBs, either being "beneficial" or "detrimental". It is well known that the presence of GBs becomes a potential barrier to dislocation motion, resulting in the generation of local stress concentration and strengthening during plastic deformation in polycrystalline solids. The strengthening by grain refinement, through the introduction of a higher density of GBs, has been extensively applied to an improvement in the strength of polycrystalline materials, up to now. For polycrystal hardening or GB strengthening, the microstructural parameter: "grain size" or "average grain size" has been used as powerful parameter associated with grain boundaries, for quantitative discussions on possible microstructural effects on the strength and fracture properties of polycrystals. The Hall-Petch relation, which is described by the linear relation between the flow stress and fracture stress and the inverse root of grain size, is a standard analysis in terms of the discipline of metallurgy and modern MSE since the middle of the 1950's [6,7]. We can easily predict higher flow stress and fracture stress for polycrystals with the finer grain size. However, there is a general recognition that the experimental data on the flow stress and fracture stress do not always follow the linear curve and deviate at smaller grain size.

In fact, the parameter "grain size" is only a geometrical one to describe the spacing between the positions of lattice discontinuity and does not mean any physical significance suggesting the structuredependent properties of GBs which have a large structural and geometrical variety and flexibility. One simple question is raised, regarding conventional and established strengthening method by grain refinement.
When the strengthening by grain refinement is applied to polycrystalline materials, even nanocrystalline materials, unfortunately the materials almost inevitably become more brittle. Why does the introduction of a higher density of GBs bring about a detrimental effect? In the authors' opinion, the strengthening by grain refinement needs to be reexamined from the view point of structure-dependent GB mechanical properties, as discussed in this paper.

In recent years, we have come to know much more about possible effects of GBs on bulk properties of polycrystalline solids than before. This is because fundamental studies of GB structure and properties have provided us with enormous new knowledge about intrinsic and extrinsic structure-dependent GB properties [8-11] and their effect on bulk properties of polycrystalline materials [12-15], over the last half century since the 1960's, after the first book on the topic of "Grain Boundaries" in metals by D. McLean [16]. Probably Wyrzykowski and Grabski were the first who gave a new insight into the Hall-Petch relation taking into consideration its dependence on the GB structure in aluminum polycrystals [17].

\section{Fracture processes in brittle metals and alloys}

\subsection{Observations of fracture processes}

In-situ observations of fracture processes can provide us with direct and useful images of fracture behavior and mechanisms in real polycrystals, as we know from "seeing is believing". We can often find some useful clue and insight into a promising solution to materials problem.

Figure 1 shows an example of in-situ observations of fracture processes in a Bi-doped coarse-grained copper specimen deformed by using a tensile stage equipped with SEM [18]. Premature grain boundary (GB) cracks were nucleated preferentially at the grain boundaries bordering coarse grains, but when propagating GB cracks meet annealing twins (indicated by A and B), the cracks will not propagate further even by much higher subsequent straining, as seen from the micrograph of fracture paths. Surprisingly, the parts of $\mathrm{A}$ and $\mathrm{B}$ at the original GBs intersecting twins never break in spite of heavy local plastic deformation. What a difference of GB nature of original long GB happened before and after the interaction with twins? Probably because the boundary character of long boundaries was locally modified by the interaction with twins, resulting in some enhancement in fracture resistance of the interacting parts of GB. Thus fracture processes can occur very heterogeneously in coarse-grained polycrystalline copper, which is well known as intrinsically ductile metal, but can be 


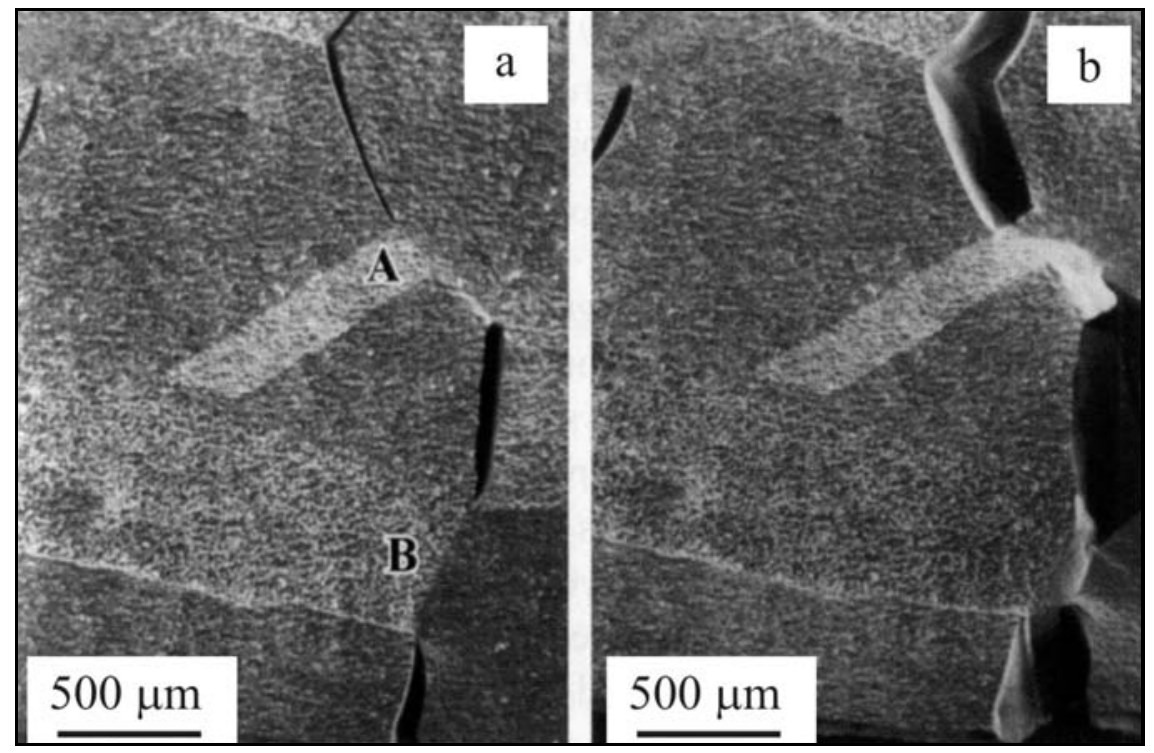

Fig. 1. In-situ observations of intergranular fracture in a polycrystal specimen of bismuth (Bi)-doped copper deformed at a tensile stage of SEM [18]. Note that crack propagation would not occur at the portions of intersection of the boundaries with twins $\mathrm{A}$ and $\mathrm{B}$.

extrinsically brittle due to doping of detrimental element bismuth $(\mathrm{Bi})$. The observations suggest that behind them there are important unknown issues which require for our insight and serious consideration into the origins of brittleness and heterogeneity of fracture processes, probably on the basis of possible effects of structure-dependent GB (intergranular) fracture processes upon bulk mechanical properties in polycrystalline materials, from microscopic and atomistic points of view as schematically shown in Fig. 2 [19].

\subsection{Structure-dependent intergranular fracture}

Recent fundamental studies of GB structure and properties have revealed that GB properties strongly depend on the type and structure of individual GBs introduced during initial processing or modified by subsequent condition. In order to study the relation of GB properties to GB type and structure, systematic experimental investigation has been extensively performed by using orientation-controlled bicrystals of metals and alloys, more recently intermetallic, semiconductor and ceramic materials. We can access fundamental data on structure-dependent GB properties in recent reference books [8-15].

It is well known that a number of GB related phenomena are involved in the fracture processes in polycrystalline solids at low and high temperatures. Recent fundamental studies of GB structure and properties have revealed that the activity or effectiveness of individual GBs as potential sites for GB-related metallurgical phenomena, including fracture, strongly depends on the GB type and structure, as shown schem-

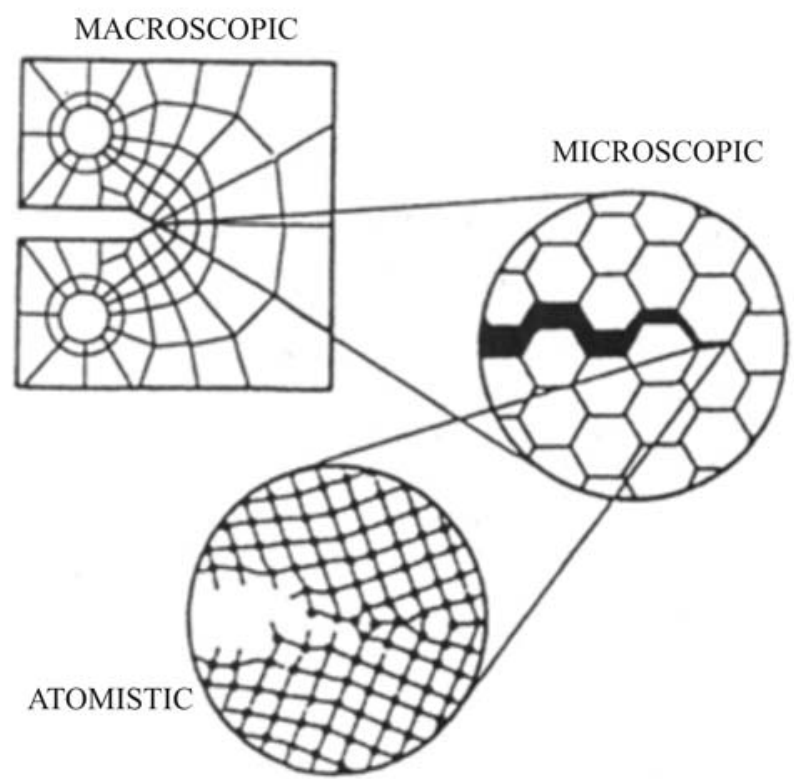

Fig. 2. Three different length scales in the study of fracture in a polycrystal [19]. Fracture studies are performed from (i) macroscopic, (ii) microscopic and (iii) atomistic scales.

atically in Fig. 3 which exhibits two different types of structure-dependent GB properties. Type A and Type $\mathrm{B}$ are characterized by the curves of the misorientation dependence, depending on their activity or effectiveness as preferential sites for GB related phenomena, either play more effective or less effective roles [20]. It is evident that some low-energy special boundaries like low-angle boundaries (with the misorientation $\left.\Theta<15^{\circ}, \Sigma 1\right)$, and high-angle/low- $\Sigma(3<\Sigma<29)$ 


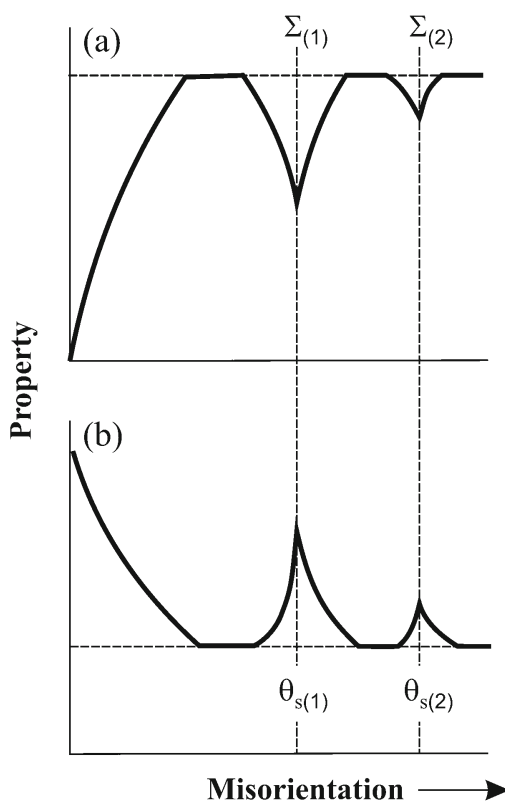

I. Type-A Boundary Properties: Curve (a)

- Grain Boundary Energy (level)

- Corrosion (width/depth)

- Segregation (amount/width, activation energy)

- Diffusion, - Migration, - Sliding (rate/activation energy)

- Hardness (level/width)

- Electrical Resistivity (level)

\section{Type-B Boundary Properties: Curve (b)}

- Migration (activation energy for middle level purity)

- Fracture (stress/strain level)

- Precipitation (nuclei density)

- Critical temperature $T_{\mathrm{c}}$ for GB phase transformation

- Critical current $J_{\mathrm{c}}$ for superconductivity

Fig. 3. Classification of structure-dependent GB properties [20]: Type A-GB properties show more significant behavior at higher energy boundaries, while Type B-GB properties show the opposite behavior, being more significant at lower-energy boundaries, particularly at low- $\Sigma$ boundaries with a special misorientation angle $\theta_{\Sigma}$.

coincidence site lattice (CSL) boundaries at $\Theta_{\Sigma(1)}$, $\Theta_{\Sigma(2)}$ corresponding to low- $\Sigma_{(1)}, \Sigma_{(2)}$, tend to show some unique GB properties, depending on the $\Sigma$ value. When GBs are characterized by the $\Sigma$ value more than 30, they are regarded as high-energy random GB not CSL-GB [21].

So far, orientation-controlled bicrystal specimens of brittle metals and alloys, e.g. molybdenum [2224], $\mathrm{Cu}-\mathrm{Sb}$ [25] and intermetallic $\mathrm{Ni}_{3} \mathrm{Al}$ [26] with systematically controlled boundary type and character were prepared and studied. Structure-dependent fracture stress and strain are normally depicted as a function of the misorientation angle for a specific type of boundary with a specific common rotation axes, say $<100>$ tilt or $<100>$ twist boundaries. Experimental works on orientation-controlled bicrystals can provide us with quantitative data on structure-dependent GB fracture. In particular, very careful experiments were performed for molybdenum bicrystals with specific type of GB such as low $<\mathrm{hkl}>$ tilt or twist boundaries. The fracture stress and fracture strain varies uniquely depending on the type and misorientation angle. There are several misorientation angles which correspond to higher fracture stress or fracture strain, or vice versa. We can determine the maximum values of fracture stress and strain or the minimum ones associated with strong or weak boundaries, respectively, from $S$ - $S$ curves obtained by experiments.

Regarding the structure-dependent GB properties, particularly of special low-energy/low- $\Sigma$ coincidence boundaries, such a popular question is often raised as how $\Sigma$ value can reflect the intrinsic GB properties. To

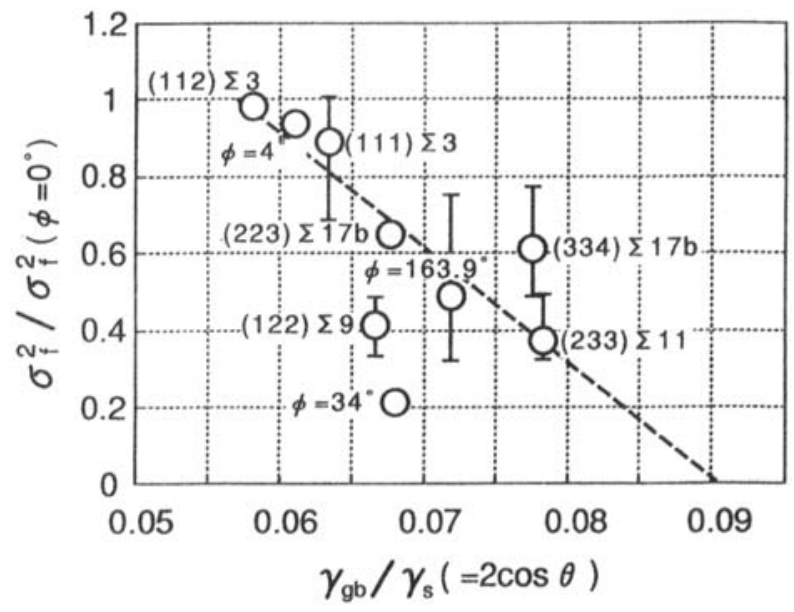

Fig. 4. Inverse dependence of the fracture stress $\sigma_{\mathrm{f}}$ on GB energy $\gamma_{\mathrm{gb}}$ for low- $\Sigma$ boundaries in molybdenum bicrystals [24]. The fracture stress $\sigma_{\mathrm{f}}$ and GB energy $\gamma_{\mathrm{gb}}$ are normalized by cleavage fracture stress $\sigma_{\mathrm{f}}(\phi=0)$ and surface energy $\gamma_{\mathrm{s}}$, respectively. The fracture stress tends to decrease with increasing GB energy and also $\Sigma$ value (except $\Sigma 11$ likely characterized as $\{110\} / /\{001\}$ plane-matching GB).

answer this question, we show the data on the fracture stress of molybdenum bicrystals with low- $\Sigma$ (from 3 to 17) coincidence boundaries. Figure 4 clearly demonstrates that the fracture stress normalized by that of single crystal systematically decreases with increasing $\Sigma$ value and the GB energy normalized by the surface energy.

This supports our general understanding that $\Sigma$ 
value can be used as a useful measure of the GB energy controlling the intrinsic nature of GB properties including GB fracture related to almost all of metallurgical phenomena. The reader can access the data on $\Sigma$-dependent fracture stress in bicrystals of metals (Mo, Al, $\mathrm{Zn})$ in the literature [27] and review paper [28].

Of particular interest and importance of general information from the schematic diagrams in Fig. 3, is that those bicrystals with higher fracture stress exhibit higher fracture strain. The observation physically means that a GB of higher fracture stress is more resistant to fracture and a larger plastic deformation must be involved in fracturing at or in vicinity of GB. The observation of higher fracture resistance can necessarily be accompanied by higher fracture strain, resulting in high fracture toughness, that is, the possibility of production of "strong and tough" polycrystal, without brittleness, if we could introduce higher-fracture-resistant boundaries, normally of low-energy. We can easily understand the intrinsic nature of structure-dependent GB fracture, from the fracture energy for GB fracture given by the following equation, $\gamma_{\mathrm{f}}=2 \gamma_{\mathrm{s}}-\gamma_{\mathrm{gb}}+\gamma_{\mathrm{p}}$, where $\gamma_{\mathrm{s}}$ and $\gamma_{\mathrm{p}}$ are the specific surface energy (strictly speaking, orientation-dependent), and the stored energy generated by plastic deformation during GB fracture, respectively. This is the basis of the prediction for the possibility of generation of "strong and tough" polycrystalline materials, leading to the new concept originally termed "grain boundary design and control" $[18,28,29]$, now "grain boundary engineering (GBE)" $[33,36]$.

\subsection{GB microstructure-dependent fracture in polycrystals}

In the case of polycrystals, the characterization of GB microstructure associated with a huge number of grain boundaries is not as simple as in bicrystals with a single boundary. We need to introduce some new parameters like the grain boundary character distribution (GBCD) and the grain boundary connectivity, in order to quantitatively characterize a specific GB microstructure existing in individual polycrystals by using a rapid and fully computerized SEM-EBSD/OIM technique developed by Adams et al. [30]. Before the advent of OIM technique, the characterization of GB microstructure in a polycrystal was carried out by very time consuming experiments with use of SEM-ECP [21] or X-ray diffraction technique.

It is a very important issue to confirm how the structure-dependent intergranular fracture observed in bicrystals can be related to bulk fracture processes and characteristics in polycrystals with a variety of microstructures. The direct evidence for this was first made by the author's group through SEM in-situ ob-

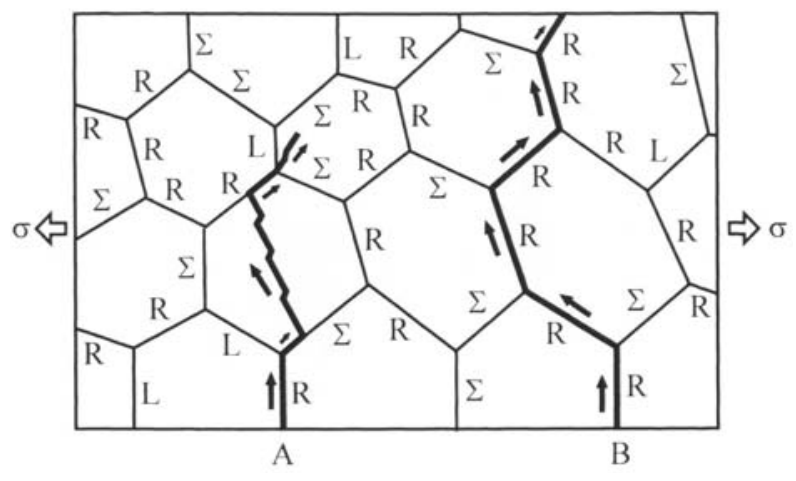

Fig. 5. Schematic diagram of GB structure-dependent fracture processes in a polycrystal [18]. Path A: combined process of intergranular fracture and transgranular fracture occurring at different types of boundaries including grain interior. Type B: typical intergranular fracture occurring continuously at connecting random $(\mathrm{R})$ boundaries.

servations of fracture processes which were carried out by using coarse-grained beta brass polycrystalline specimens whose GB microstructures were already characterized before fracture test [31]. GB microstructures were characterized by using then newly developed SEM-ECP technique and extrinsically brittled by liquid gallium to induce brittle fracture before fracture test. It was found that cracks nucleated preferentially at high-energy random boundary and propagated further to other connecting random boundaries, resulting in the dominant occurrence of intergranular fracture leading to a typical intergranular brittleness, as indicated by the Path B in the schematic figure of fracture processes in a polycrystal originally depicted in [18] and shown in Fig. 5. On the other hand, as clearly seen from the Path A, when a propagating crack meets low-energy fracture resistant boundary such as low- $\Sigma$ coincidence boundaries $(\Sigma<29)$, at a triple junction cannot propagate from high-energy random to lowenergy coincidence boundaries due to a large difference in their intrinsic fracture stress.

From the in-situ observations of the fracture behavior in beta brass polycrystals, it is revealed that the fracture mode can change from intergranular to transgranular fracture, or vice versa, depending on the frequency of specific types of boundaries and manner of interaction between different types of boundaries, now defined in some detail as "grain boundary microstructure". In the case of transgranular fracture where the propagating crack can proceed in transgranular manner involving some plastic deformation in the grain interior, there must be the intersection with low-energy/fracture resistant boundary. Since the involvement of plastic deformation during fracturing in crystalline solid needs the plastic energy much higher than the grain boundary energy, the fracture Path A suggests the strategy for control of intergranular frac- 

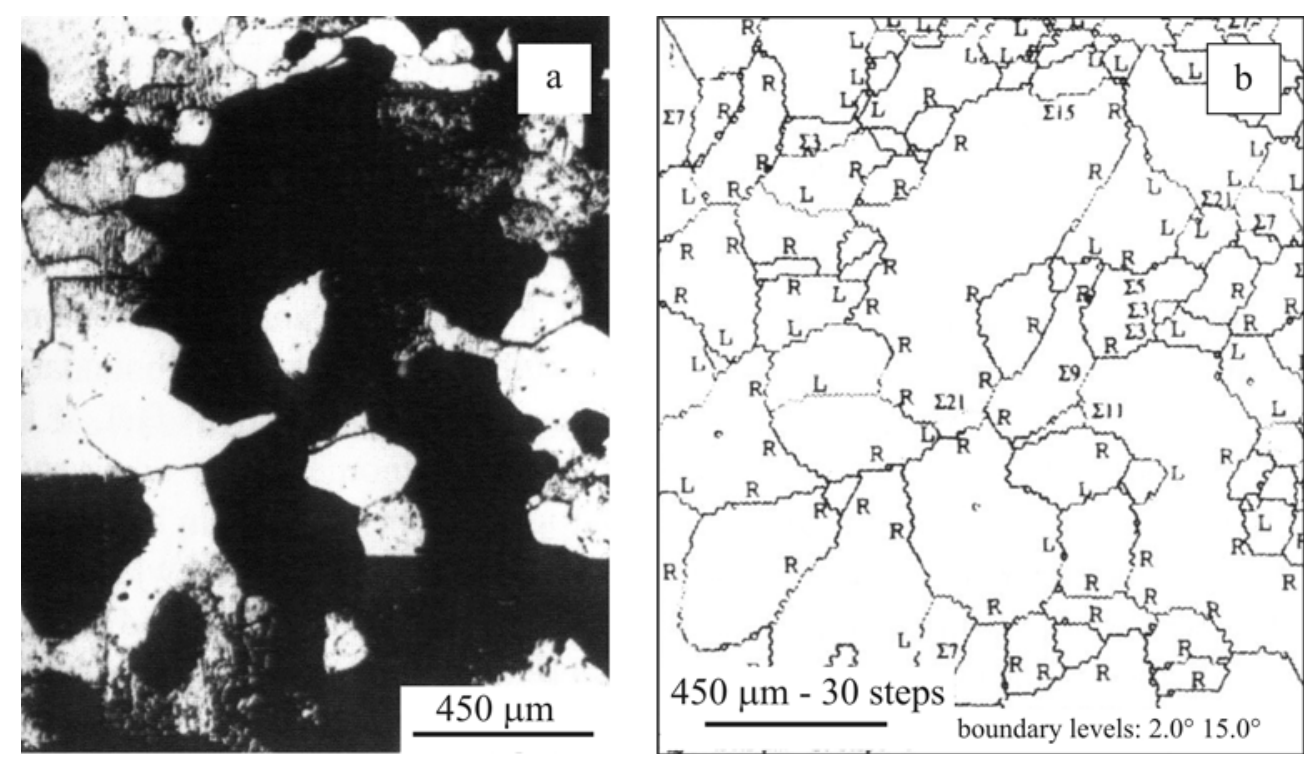

Fig. 6. An example of the characterization of GB microstructure by SEM-EBSD/OIM system for a molybdenum polycrystal produced by thermomechanical processing of a single crystal of (111) initial orientation [27]. (a) Optical micrograph, (b) GB characterization by OIM.

ture and intergranular brittleness in brittle materials. It is not difficult to suggest that different fracture processes would occur for polycrystals with different GB microstructures produced by applying some suitable processing route using different processing conditions to introduce optimal GB microstructure.

Now we have obtained fundamental knowledge about the possibility of toughening of brittle polycrystalline materials through the control of structuredependent intergranular fracture by introducing more low-energy/fracture resistant boundaries in polycrystals. This is the strategy for our goal to "Strong and Tough Materials" given by the concept of "Grain Boundary Design and Control" or Grain Boundary Engineering (GBE).

\section{GBE approach toward "strong and tough" materials}

Since the concept of "Grain Boundary Design and Control" or "Grain Boundary Engineering (GBE)" was proposed, the control of intergranular fracture had been extensively attempted for different kinds of intrinsically or extrinsically brittle materials. The studied materials are of the first category of intrinsically brittle ones like refractory metals (Mo), intermetallics $\left(\mathrm{Ni}_{3} \mathrm{Al}\right)$, ceramics $(\mathrm{SiC})$, and of the second category which are inherently ductile but extrinsically embrittled by segregation of detrimental elements, liquid metal, and oxidation. We could successfully apply GBE to controlling severe intergranular brittleness in all kinds of the studied materials. The achievements of on-going progress in GBE have been reported by
Watanabe and co-workers almost regularly in research review [32], papers [33, 34] and recent books [35, 36].

\subsection{Structural heterogeneity of $G B$ microstructures}

It has been theoretically predicted [27] and experimentally proved that the "grain boundary microstructure", which is composed of grain boundaries of different types and various geometrical configurations, evidently controls the fracture processes and fracture characteristics of polycrystals. Of particular importance is that the observed heterogeneity of fracture processes was found to originate from the heterogeneity of GB microstructure existing in polycrystals even with a uniform and sharp grain size distribution. At present, GB microstructure can be quantitatively defined and characterized by SEM-EBSD/OIM technique for almost all kinds of polycrystalline materials of different crystal structures (bcc, fcc, hcp, even non-cubic crystal) and of various grain sizes ranging from micron to nanometer order.

The two important parameters, i.e. the grain boundary character distribution (GBCD) and the grain boundary connectivity, have been generally used to quantitatively characterize GB microstructures [18]. An example of the characterization of GB microstructure in a molybdenum polycrystal produced by thermomechanical processing of a single crystal is shown in Fig. 6. There is a general tendency that larger grains are surrounded by random boundaries $(\mathrm{R})$ while smaller grains by low-angle boundaries (L) or low- $\Sigma$ coincidence boundaries. This is reasonably explained by the relation between GBCD and grain size 


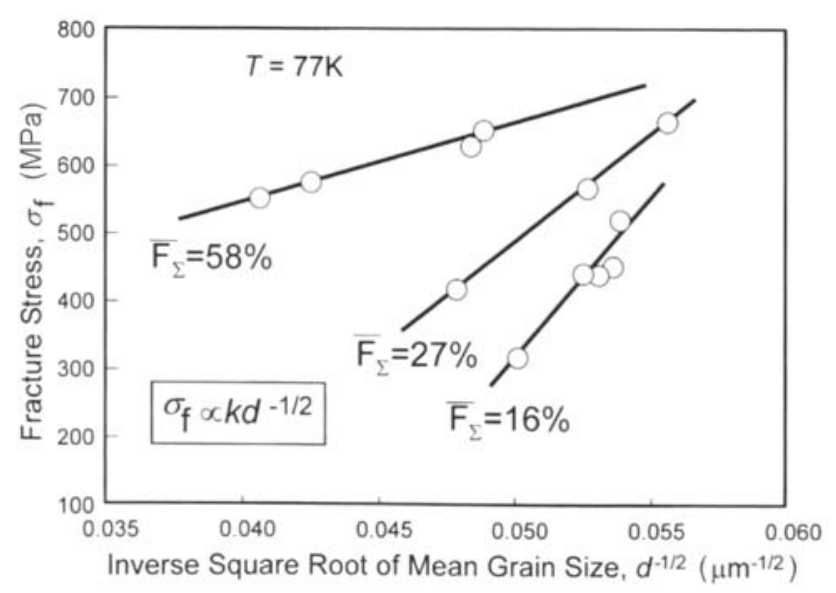

Fig. 7. The Hall-Petch type plot of the fracture stress vs. the mean grain size, for three groups of polycrystalline molybdenum specimens with different levels of the fraction of low- $\Sigma$ coincidence boundaries $F_{\Sigma}$ [39].

observed for ordinary polycrystals of metals produced by thermomechanical processing involving recrystallization and grain growth in deformed sample. The fraction of random boundaries tends to increase during grain growth because of a higher mobility of random boundary [37]. During recrystallization and grain growth, initially formed low-energy boundaries are transformed into GB of higher energy and mobility, finally to random boundaries. Quite recently Kobayashi et al. have performed a systematic study of GB structures in molybdenum polycrystals produced by thermomechanical processing of single crystals of different initial orientations [38]. It was found that a systematic change of the frequency of triple junction can occur in association with the evolution of sharp/different types of texture. This observation can provide us with systematic changes of the grain boundary connectivity as a function of low-energy boundaries such as low-angle and low- $\Sigma$ boundaries. It was also found that a systematic increase of low-energy boundaries brings about the evolution of special types of triple junction composed of three low-energy boundaries which are resistant to crack propagation in a polycrystal.

\subsection{Toughening of molybdenum by GBE}

Now let us look at an example of toughening of intrinsically brittle molybdenum polycrystals by GBE, taken from our previous work [39]. Figure 7 shows the grain size dependence of the fracture stress for molybdenum polycrystals with different GB microstructures. The fracture stress increases with decreasing grain size on the Hall-Petch relation. Of particular interest is that the level of the fracture stress and the slope of the curves depend on the fraction of low- $\Sigma$ boundaries $F_{\Sigma}$. It is evident that GB mi- crostructure with $F_{\Sigma}$ brings about higher fracture stress. The observed decrease of the Hall-Petch slope $k$ was suggested to be caused by a change of dominant fracture mechanism, from crack nucleation to crack propagation with decreasing the fraction of fracture resistant/low- $\Sigma$ boundaries, $F_{\Sigma}$. Thus it was proved that toughening by GBE was successfully applied to intrinsically brittle molybdenum polycrystals.

\subsection{Fractal analysis of GB microstructure for fracture control}

Until recently, GBE for fracture control has been undertaken by controlling the GB microstructure mainly focusing GBCD by applying some suitable materials processing to produce the optimal GBCD for desirable mechanical properties such as strength or ductility, corrosion resistance, and others. It is suggested that the conventional approach of GBE is not sufficient because the interrelation between GBCD, the grain boundary connectivity and crack paths has not been quantitatively and fully evaluated. Therefore, the conventional GBE for fracture control needs to be refined by taking "fractal analysis" for the connectivity of possible crack paths in relation to GBCD and grain boundary connectivity, on the basis of the result of GB microstructure analysis. The most recent work of GBE for fracture control for brittle materials was performed by Kobayashi et al. [40] by taking "Fractal Analysis" of GB microstructure. In this work, the fractal analysis of GB microstructure was carried out for the control of segregation-induced intergranular fracture in extrinsically brittled/sulfur-doped polycrystalline nickel. The fractal dimension of grain boundary network was analyzed by the box-counting method from the trace of the random boundary network with the maximum connectivity, or the maximum random boundary connectivity (MRBC) which can become a propagation path of cracks. Further detail of the fractal analysis of GB microstructure should be referred to the original paper. It was found that there was an excellent relation between the fractal dimension of the MRBC, $D_{\mathrm{R}}$, and the fraction of low- $\Sigma$ boundaries $F_{\Sigma}$. A change of fracture mode from brittle intergranular fracture to ductile transgranular fracture was predicted to occur at the fraction of low- $\Sigma$ boundaries $F_{\Sigma}=60 \%$, corresponding to the threshold value predicted by the theory of percolation processes occurring in GB network in polycrystals. Probably this new approach may bring about a more precise and effective application of GBE for "strong and tough materials" in the future. To our present knowledge, theoretical and experimental works have been performed on the fracture processes for model and real polycrystalline materials based on "fractal analysis", for example by Tanaka et al. [41]. However, until recently, there was no work based on "fractal analyses" of possible fracture paths for inter- 


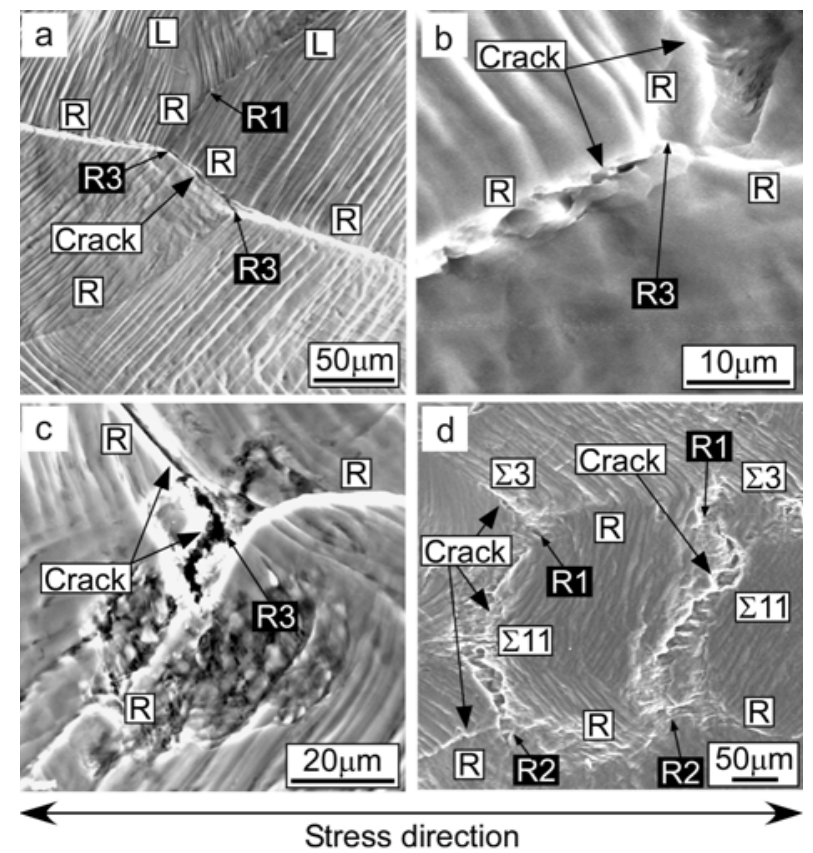

Fig. 8. SEM micrographs of fatigue intergranular cracks nucleated and propagated at different types of GBs in coarse-grained aluminum polycrystals [42].

granular or transgranular fracture in connection with GB microstructures in real polycrystalline materials. This may bring a new step or breakthrough toward future fracture control and development of strong and tough materials.

\section{GBE for enhanced fatigue strength and fracture resistance}

Finally, we would like to present our recent achievements of GBE toward the enhancement in fatigue strength and fatigue fracture resistance at this anniversary symposium. This may be one of key issues in the research area of fatigue deformation and fracture, in which Prof. Pavel Lukac and co-workers have been deeply involved and greatly contributed to our current understanding of fatigue fracture properties of engineering materials. Quite recently we have performed a systematic study of GBE toward the improvement in fatigue strength and fracture resistance for ordinary ductile polycrystalline aluminum [42] and austenitic stainless steel $[43,44]$, and extrinsically brittled coarse-and fine-grained Ni-S alloy [45] and electrodeposited nanocrystalline Ni-P alloy $[46,47]$ with systematically controlled GB microstructures and a large variety of the average grain size ranging from micrometer to nanometer order.

Let us briefly look at some new findings from the recent works on GBE for enhanced fatigue strength
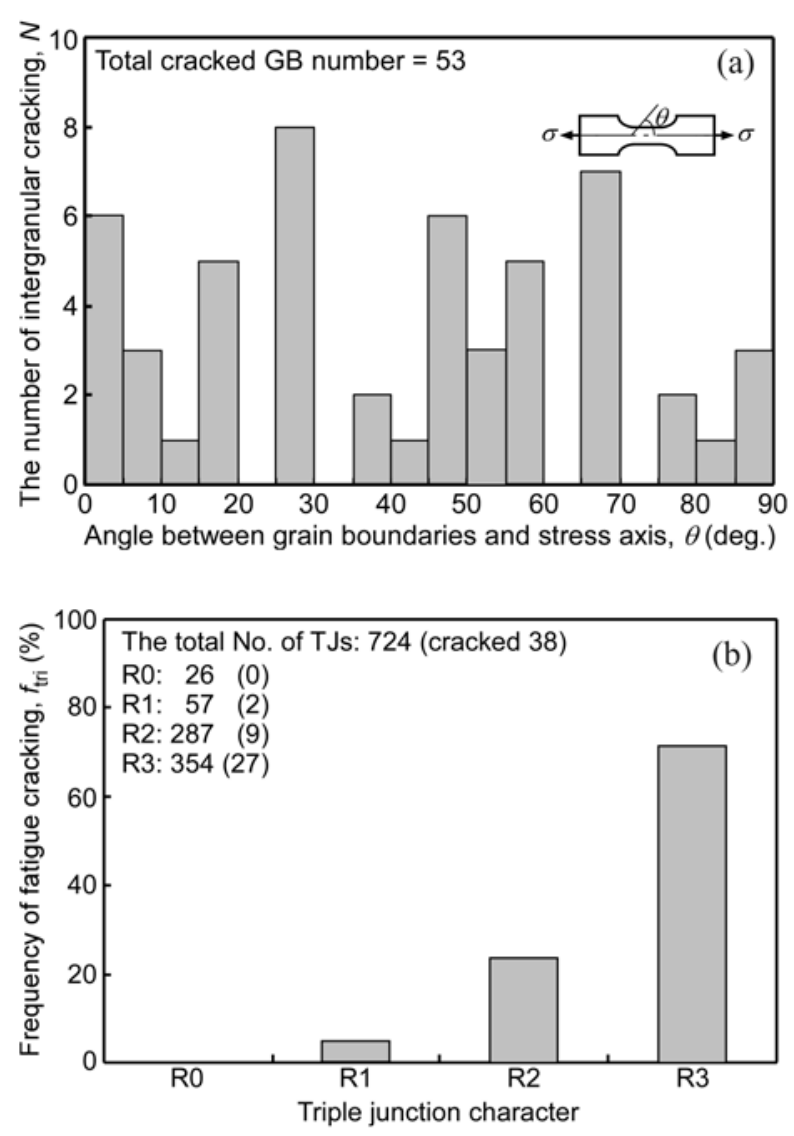

Fig. 9. Effects of GB inclination angle $\theta$ to the tensile and triple junction character on fatigue intergranular fracture in coarse-grained aluminum [42].

and fatigue fracture resistance for the metallic materials mentioned above. The effects of the character of GB and triple-junction were studied on integranular fatigue crack nucleation in coarse-grained aluminum specimens [42]. Figures 8a-d show SEM micrographs of intergranular cracks observed on the surface of a specimen fracture by fatigue deformation at a stress amplitude of $15.5 \mathrm{MPa}$ at a frequency of $10 \mathrm{~Hz}$. The characters of individual GBs and triple-junctions are denoted by capital letters, respectively for low-angle, low- $\Sigma$ CSL and random boundaries by $L, \Sigma$ with numeral, and R. The characterization of triple-junctions was made by the number of random boundaries meeting at individual junctions, as R3, R2, R1. Sharp intergranular cracks were observed to preferentially nucleate at random boundaries, but sometimes at $\Sigma 3$ and $\Sigma 11$ CSL boundaries indicating heavy local plastic deformation, as seen in Fig. 8d. As for the effect of the inclination of GBs on crack nucleation, Fig. 9a shows an interesting finding on integranular crack nucleation. The number of intergranular cracks was counted as a function of the angle $\theta$ between the traces of GBs and the stress direction, as indicated by the 


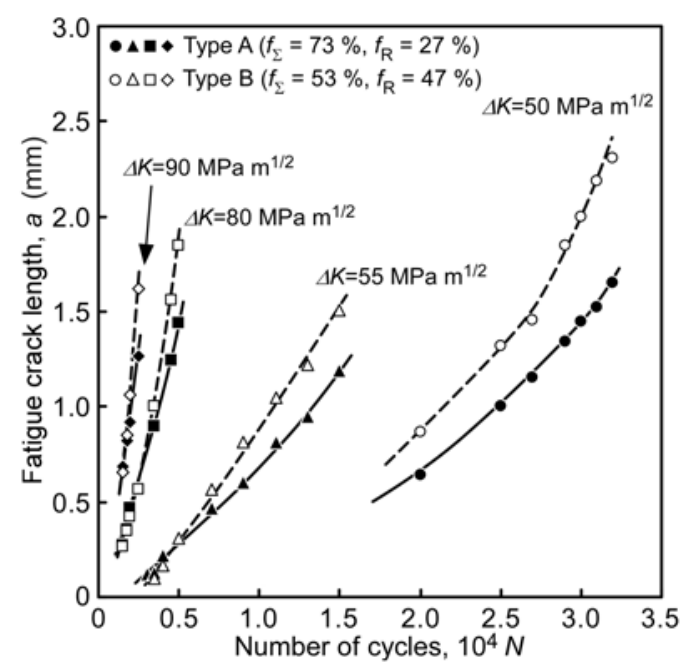

Fig. 10. Relationship between the total length of fatigue cracks and the number of cycles on different levels of the stress intensity, for the two types of austenitic stainless steel specimens Type A and Type B with different fractions of low- $\Sigma$ coincidence boundaries or random boundaries [44].

inset. It is evident that there is no significant dependence of occurrence of intergranular fatigue cracking on the inclination of GBs. On the other hand, as clearly seen from Fig. 9b, the presence of random boundaries and of the triple-junctions composed more random boundaries is the most important factor controlling crack nucleation in aluminum polycrystal under cyclic deformation. The findings obtained from this work clearly demonstrate the dominant effects of the character of GBs and triple-junctions on intergranular fatigue cracking in ductile aluminum polycrystals.

Next, let us introduce an interesting finding on fatigue crack propagation in austenitic stainless steel $[43,44]$. Figure 10 shows the experimental result on crack propagation behavior for the two types of specimens with different GB microstructures specified by different values of the fraction of low- $\Sigma$ or random boundaries (R). As is clear from the curves on which the fatigue crack length was plotted as a function of the number of cycles, $N$, the crack propagation rate was faster for Type B specimens of a higher fraction $(47 \%)$ of random boundaries than Type A $(27 \%)$, conversely, of a lower fraction $(53 \%)$ of fracture resistant low- $\Sigma$ boundaries for the former than the latter (73\%). Thus it is evident that the GB microstructure of a higher fraction of strong/low- $\Sigma$ GBs can bring about a significant enhancement of the resistance to intergranular crack propagation which can be the primary origin of brittle fatigue fracture due to intergranular fracture in metallic materials even if they are in the category of ductile materials.

Finally, let us discuss the applicability of GBE for controlling the brittleness due to intergranular fracture, on the basis of our most recent work on GBE for brittle fracture control in extrinsically brittle sulfurdoped nickel under cyclic deformation [45]. It is well known that the segregation of sulfur to grain boundaries brittles intrinsically ductile nickel polycrystals.

Segregation-induced intergranular brittleness is a serious problem in development of high performance nickel base alloys used for their service at high temperatures under cyclic loading.

We show SEM micrographs of fracture surfaces taken from the two different specimens prepared by different thermomechanical processing routs to introduce different GB microstructures. Type A specimen had the fraction of low- $\Sigma$ GBs $\left(F_{\Sigma}\right) 53 \%$ and the average grain size $d=45 \mu \mathrm{m}$, and Type B had $F_{\Sigma}=40 \%$ and $d=69$. As shown in Fig. 11, Type A specimen shows a typical ductile fracture surface having many dimple patterns, while Type B specimen shows a typical intergranular fracture surfaces composed of flat GB facets. It is surprising to see that the difference of the fraction of low- $\Sigma$ GBs $\left(F_{\Sigma}\right)$ by $13 \%$ brings about a change of fracture mode from transgranular ductile fracture to intergranular brittle fracture, for Type A and Type B. It should be noted that an increase of the fraction of $F_{\Sigma}$ was found to be very effective even if small as about $10 \%$.

A similar significant effect of an increase of the fraction of $F_{\Sigma}$ on toughening through intergranular fracture control was found in sulfur-doped nanocrystalline nickel produced by electrodeposition [46]. An increase of $F_{\Sigma}$ only by $9 \%$ brought about an increase in the fracture toughness more than twice from $K_{\mathrm{IC}}=$ 1.1 to $2.5 \mathrm{MPa} \mathrm{m}^{1 / 2}$ in nanocrystalline materials with a much higher density of grain boundaries than that of an ordinary polycrystalline material. Moreover, it was recently observed that significant grain growth takes place and enhances intergranular fracture during cyclic deformation in nanocrystalline materials even at room temperature [47]. Our understanding of possible mechanisms of grain growth-assisted intergranular fracture and the strategy of the control of intergranular fracture is still premature and awaits the right time of challenging GBE toward the development of high performance nanocrystalline materials [48] for their service as machine components under static and cycling loading conditions.

\section{Conclusions}

A long pending dilemma, "strong and brittle" materials has been revisited. A new concept of Grain Boundary Engineering (GBE) proposed in the early 1980s has been extensively applied to the toughening of different types of brittle materials in recent years, drawing an increasing interest of researchers. This art- 

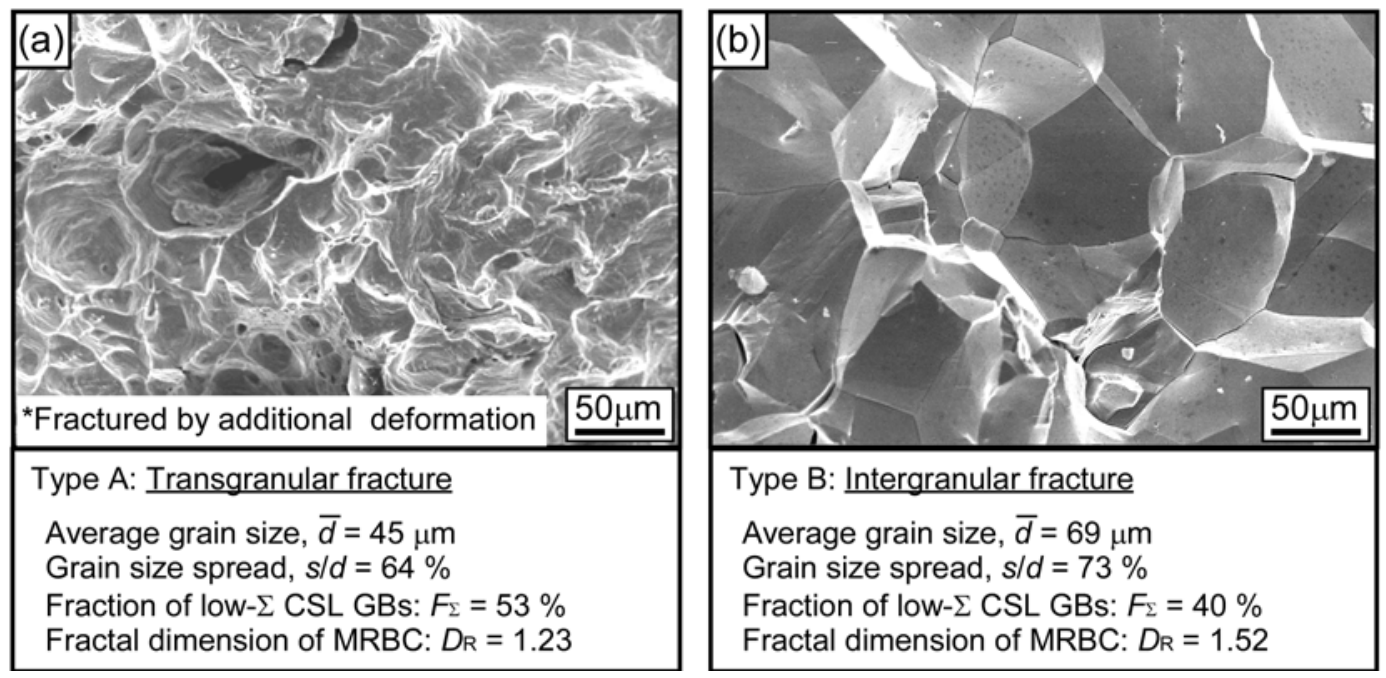

Fig. 11. SEM micrographs of typical fracture surface produced by static bending test in sulfur-doped nickel polycrystals [45]. Type A: typical transgranular fracture, Type B: typical integranular fracture, depending on the fraction of low- $\Sigma$ coincidence boundaries $F_{\Sigma}$.

icle has shown the most recent progress in GBE for controlling brittleness in intrinsically and extrinsically brittle polycrystalline metallic and intermetallic materials. It has been confirmed that brittle materials can be transformed into "Strong and Tough" materials by GBE through controlling the grain boundary microstructures, which generate higher fracture stress and higher fracture toughness than those of conventional polycrystalline materials, even in nanocrystalline materials of an extremely high density of grain boundaries.

\section{Acknowledgements}

This paper is dedicated to Prof. Pavel Lukac on his $80^{\text {th }}$ birthday anniversary.

The authors would like to thank their coworkers, especially Prof. Hiroyuki Kokawa and Prof. Sadahiro Tsurekawa, for their deep involvement in GBE project during the last three decades. Financial supports from JSPS and generous research foundations are greatly appreciated which much helped our GBE works on structural and functional materials. One (T. W.) of the authors wishes to express his particular gratitude to Prof. Zuo Liang and Prof. Zhao Xiang, of Northeastern University, China and Prof. Claude Esling of Universite de Lorraine, France for their collaboration for many years.

\section{References}

[1] Reid, C. N.: J. Less-Common Metals, 9, 1965, p. 105. doi:10.1016/0022-5088(65)90088-3

[2] Low, J. R.: Trans. Met. Soc. AIME, 245, 1969, p. 2481.

[3] Hahn, G. T.: Met. Trans A, 15, 1984, p. 947. doi:10.1007/BF02644685
[4] Thompson, A. W., Knott, J. F.: Met. Trans. A, 24, 1993, p. 523. doi:10.1007/BF02656622

[5] Gumbsch, P., Cannon, R. M.: MRS Bulletin, 25, 2000, p. 15 doi: $10.1557 / \mathrm{mrs} 2000.68$

[6] Baker, T. N. (ed): Yield, Flow and Fracture of Polycrystals. London, Appl. Sci. Pub. 1983.

[7] Otooni, M. A., Armstrong, R. W., Grant, N. J., Ishizaki, K. (eds.): Grain Size and Mechanical PropertiesFundamentals and Applications. Pittsburgh, MRS 1995.

[8] Gleiter, H., Chalmers, B.: High-Angle Grain Boundaries: Progress in Materials Science. Oxford, Pergamon Press 1972.

[9] Chadwick, G. A., Smith, D. A. (eds.): Grain Boundary Structure and Properties. London, Academic Press 1976.

[10] Wolf, D., Yip, S.: Materials Interfaces - Atomic-Level Structure and Properties. London, Chapman \& Hall 1992.

[11] Sutton, A. P., Balluffi, R. W.: Interfaces in Crystalline Materials. Oxford, Oxford Sci. Pub. 1995.

[12] Gottstein, G., Shvindlerman, L. S.: Grain Boundary Migration in Metals: Thermo-Dynamics, Kinetics, Applications. Boca Raton, CRC Press 1999.

[13] Ray, R. K., Murthy, V. S. R., Batra, N. K., Padmanabhan, K. A., Ranganathan, S. (eds.): Materials for the Third Millennium. New Delhi, Oxford \& IBH Pub. 2001.

[14] Lejcek, P.: Grain Boundary Segregation in Metals. Berlin, Heidelberg, Springer 2010. doi:10.1007/978-3-642-12505-8

[15] Priester, L. (ed.): Grain Boundaries from Theory to Engineering. Dordrecht, Springer 2013. doi:10.1007/978-94-007-4969-6

[16] McLean, D.: Grain Boundaries in Metals. London, Oxford University Press 1957.

[17] Wyrzykowski, J. W., Grabski, M. W.: Phil. Mag. A, 53, 1986, p. 505. doi:10.1080/01418618608242849

[18] Watanabe, T.: Res Mechanica, 11, 1984, p. 47.

[19] Ohr, M. (ed.): Abstract Book of the 2nd International 
Conf. on Fundamentals of Fracture, Riverside Motor Lodge, Gatlinburg, Tennessee, USA, Oak Ridge National Laboratory 1985.

[20] Watanabe, T.: J. Mater. Sci., 46, 2011, p. 4095. doi:10.1007/s10853-011-5393-z

[21] Watanabe, T., Fujii, H., Oikawa, H., Arai, K. I.: Acta Met., 37, 1989, p. 941. doi:10.1016/0001-6160(89)90021-7

[22] Brosse, J. S, Fillit, R., Biscondi, M.: Scripta Met., 15, 1981, p. 619.

[23] Kurishita, H., Kuba, S., Kubo, H., Yoshinaga, H.: Trans. JIM, 26, 1985, p. 332.

[24] Tsurekawa, S., Tanaka, T., Yoshinaga, H.: Mater. Sci. Eng. A, 176, 1994, p. 341. doi:10.1016/0921-5093(94)90997-0

[25] Monzen, R., Masuda O., Miura, H.: Mater. Sci. Eng. A, 387-389, 2004, p. 424. doi:10.1016/j.msea.2004.05.036

[26] Su, J.-Q., Denuma, M., Hirano, T.: Phil. Mag. A, 82, 2002, p. 1541.

[27] Lim, L. C., Watanabe, T.: Acta Met. Mater., 38, 1990, p. 2507. doi:10.1016/0956-7151(90)90262-F

[28] Watanabe, T.: Mater. Sci. Eng. A, 176, 1994, p. 39. doi:10.1016/0921-5093(94)90957-1

[29] Watanabe, T.: Mater. Sci. Eng. A, 166, 1993, p. 11. doi:10.1016/0921-5093(93)90306-Y

[30] Adams, B. L., Wright, S. I., Kunze, K.: Met. Trans. A, 24, 1993, p. 819. doi:10.1007/BF02656503

[31] Watanabe, T., Tanaka, M., Karashima, S.: Embrittlement by Liquid and Solid Metals. Ed.: Kamdar, M. H. Warendale, TMS-AIME 1984.

[32] Watanabe, T.: Mater. Sci. Eng. A, 176, 1994, p. 39. doi:10.1016/0921-5093(94)90957-1

[33] Watanabe, T., Tsurekawa, S.: Acta Mater., 47, 1999, p. 4171. doi:10.1016/S1359-6454(99)00275-X

[34] Watanabe, T., Tsurekawa, S.: Mater. Sci. Eng. A, 387389, 2004, p. 447. doi:10.1016/S0921-5093(04)00653-7
[35] Watanabe, T., Tsurekawa, S., Zhao, X., Zuo, L.: Microstructure and Texture in Steels and Other Materials. London, Springer 2009. doi:10.1007/978-1-84882-454-6_4

[36] Watanabe, T.: Microstructural Design of Advanced Engineering Materials. Ed.: Molodov, D. A. Weinheim, Wiley-VCH 2013. doi:10.1002/9783527652815

[37] Watanabe, T.: Scripta Met., 21, 1987, p. 427. doi:10.1016/0036-9748(87)90174-8

[38] Kobayashi, S., Tsurekawa, S., Watanabe, T., Kobylanski, A.: Phil. Mag., 88, 2008, p. 489. doi:10.1080/14786430801891748

[39] Tsurekawa, S., Watanabe, T.: MRS Symp. Proc., 586, 2000 , p. 237.

[40] Kobayashi, S., Maruyama, T., Tsurekawa, S., Watanabe, T.: Acta Mater., 60, 2012, p. 6200. doi:10.1016/j.actamat.2012.07.065

[41] Tanaka, M., Kato, R., Kayama, A. J.: Mater. Sci., 37, 2002, p. 3945. doi:10.1023/A:1019676027293

[42] Kobayashi, S., Inomata, T., Kobayashi, H., Tsurekawa, S., Watanabe, T.: J. Mater. Sci., 43, 2008, p. 3792. doi:10.1007/s10853-007-2236-z

[43] Kobayashi, S., Hirata, M., Tsurekawa, S., Watanabe, T.: Procedia Engineering, 10, 2011, p. 112. doi:10.1016/j.proeng.2011.04.021

[44] Kobayashi, S., Nakamura, M., Tsurekawa, S., Watanabe, T.: J. Mater. Sci., 46, 2011, p. 4254. doi:10.1007/s10853-010-5238-1

[45] Kobayashi, S., Maruyama, T., Saito, S., Tsurekawa, S., Watanabe, T.: J. Mater. Sci., 49, 2014, p. 4007. doi:10.1007/s10853-014-8056-z

[46] Kobayashi, S., Tsurekawa, S., Watanabe, T., Palumbo, G.: Scripta Mater., 62, 2010, p. 294. doi:10.1016/j.scriptamat.2009.11.022

[47] An, X. H., Wu, S. D., Wang, Z. G., Zhang, Z. F.: Acta Mater., 74, 2014, p. 200. doi:10.1016/j.actamat.2014.04.053

[48] Kobayashi, S., Kamata, A., Watanabe, T.: Acta Mater., 91, 2015, p. 70. doi:10.1016/j.actamat.2015.03.028 